\title{
Two stream hypothesis of visual processing for navigation in mouse
}

\author{
Aman B Saleem \\ UCL Institute of Behavioural Neurosciences, Department of Experimental Psychology, University \\ College London, London, WC1H 0AP, UK; aman.saleem@ucl.ac.uk
}

\begin{abstract}
Vision research has traditionally been studied in stationary subjects observing stimuli, and rarely during navigation. Recent research using virtual reality environments for mice has revealed that responses even in the primary visual cortex are modulated by spatial context identical scenes presented in different positions of a room can elicit different responses. Here, we review these results and discuss how information from visual areas can reach navigational areas of the brain. Based on the observation that mouse higher visual areas cover different parts of the visual field, we propose that spatial signals are processed along two-streams based on visual field coverage. Specifically, this hypothesis suggests that landmark related signals are processed by areas biased to the central field, and self-motion related signals are processed by areas biased to the peripheral field.
\end{abstract}

\section{Highlights}

1. Spatial context modulates responses in the visual cortex during navigation

2. Mouse higher visual areas show a bias in their coverage of the visual field

3. We hypothesize that bias in visual field coverage relates to functional specialisation

4. Landmark signals are likely processed by areas biased to the central visual field

5. Self-motion signals are likely processed by areas biased to the peripheral visual field

\section{Introduction}

Navigation is one of the fundamental requirements for survival as it is needed to find food, approach potential mates, or reach safety when there is a threat. To navigate, one needs to observe and interpret the surrounding environment, for which vision is key in most mammals, from humans to rodents. As humans, we use visual information to understand our surroundings, remember them, and direct our future movements. Similarly, rodents commonly use vision for navigation, which is demonstrated by classic tests of spatial memory. For example, in the Morris water maze task - one of the most popular tests of spatial memory in rodents - animals use distal visual cues to navigate their environment [1]. Consistent with this, vision is known to strongly influence neural representations of space: for instance, place cells (in hippocampus) and head direction cells (in various brain regions) rotate their representation of space when visual features in the environment are rotated [27]. Yet, how visual information reaches the navigational system remains unknown as few studies have measured activity in the visual system during navigation $[8,9]$. This has mainly been due to the difficulty of controlling visual stimulation in freely moving animals. Fortunately, the recent development of the rodent virtual reality systems [10,11] has removed this constraint, and expanded the possibilities for studying vision during navigation 
$[12,13]$. In these systems, a head-fixed animal can explore a virtual environment by running on a wheel or ball. Using such rodent virtual reality in combination with high-resolution imaging or multi-electrode array recordings, there have been an increasing number of studies investigating the mouse visual system during navigation [14-20]. In this article, we will review recent findings regarding how navigation alters responses in visual areas. This new data provides new challenges to existing theories of visual processing and an opportunity to revisit them from a fresh perspective of navigation. We propose a new twostream hypothesis - that visual information for navigation is processed along two streams that are based on visual field coverage.

\section{Overview of mouse visual cortex}

We first provide a very brief overview of the mouse visual system (see Glickfeld \& Olsen [21] for a detailed review). Mice have a large field of view, covering a range of over $270^{\circ}$ along the horizontal axis and over $120^{\circ}$ along the vertical axis [22,23]. Similar to other mammalian visual systems, retinal cells of mouse project to two brain regions, the superior colliculus in the mid-brain and the dorsal lateral geniculate nucleus (LGN) in the thalamus, and LGN neurons in turn project mainly to the primary visual cortex (V1) (Figure 1A). Inputs into V1 are retinotopically organised, i.e. there is a topographic progression of the part of the visual field that elicits a response in the region. Using the presence of retinotopic maps, and anatomical connectivity with $\mathrm{V} 1,11$ distinct higher visual areas have been identified in mouse [24-26]. These areas surround V1 and are named based on their topographic position relative to $\mathrm{V} 1$, and include (clockwise from left): lateromedial (LM), laterointermediate $(\mathrm{Li})$, anterolateral $(A L)$, rostrolateral $(R L)$, anterior $(A)$, anteromedial $(A M)$, posteromedial (PM), posterior (P) and postrhinal (POR) [25-27] (Figure 1B).

Subsequent to the identification of 11 distinct visual areas in mouse cortex, there has been considerable interest in understanding their functional organisation. In primates, visual areas are classically split into two streams of processing, the dorsal (where) stream for movement information and the ventral (what) stream for object identification [28,29]. Similar attempts have been made in mouse, the most influential of which is from the Burkhalter group that is based on anatomical connectivity [24,30]. They place areas LM, Li, P and POR along a ventral stream, based on their connectivity to entorhinal cortex and temporal association areas. The dorsal stream includes $A L, R L, P M, A M$ and $A$, and is based on connectivity with the retrosplenial, anterior cingulate and motor cortices [30]. The functional specialization of mouse higher visual areas have been investigated based on preferences for specific spatial and temporal frequencies, or selectivity to global motion [31-38]. To date, however, there has been limited success in understanding how functional specialisation of different mouse visual areas supports behaviour. Indeed, an exhaustive survey of responses of visual cortical neurons to various visual stimuli found that a large fraction of neurons do not even respond to classical visual stimuli such as drifting gratings [38]. We propose that functional specialisation might be better understood using a framework based on navigational requirements. 


\section{Evidence of spatial signals in mouse visual cortex}

One of the first studies to measure visual cortical activity in animals navigating a spatial environment was by Ji and Wilson [39]. They found neurons that exhibited localised firing fields, resembling place fields, in rats running through a figure-of-eight maze. However, as these observations (and similar studies [40]) were from animals exploring real environments with limited control of visual scenes, the location specificity may be due to differences in the retinal image experienced by the animals.

In order to test if activity in visual cortex is indeed modulated by spatial information, we need to create an environment in which we have precise control over the retinal images experienced by an animal. Saleem et al. [14], took advantage of a virtual reality setup for rodents $[10,11,41]$ to create such an environment - a linear corridor with two visually matching segments (Figure 1C-D). Visually matching segments have a simple prediction: if responses are driven purely by visual scenes, responses should be identical in the two matching segments of the environment.

Surprisingly, many neurons in V1 did not show such identical responses, thus suggesting responses were modulated by non-visual signals (Figure 1E). The modulation was so strong that some neurons had just a single peak in the environment (Figure 1E). This spatial modulation was much higher than what was expected by chance in visual neurons, and could not be explained by any other non-visual signals, including running speed, reward, eye movements and pupil size [14]. Similar spatial modulation of visual response has also been shown to persist even in the absence of visual cues, at least when related to reward position $[20,42]$. Thus, neurons in V1 show a strong modulation of responses by spatial context.

Is the spatial context causing this modulation of visual signals related to the signals encoded in navigational areas of the brain, i.e. the spatial signals? Saleem et al. recorded simultaneously from V1 and the CA1 region of the hippocampus using multi-electrode arrays (Figure 2A) [14]. Neural populations in both areas represented the environment, and using a Bayesian decoder, they could effectively decode position of the animal based on population activity (Figure 2B). More importantly, the position decoded from both these areas was highly correlated, including common errors, suggesting that V1 and CA1 were indeed representing the same spatial information $[14,15,40]$.

We have so far discussed how spatial signals influence responses in $\mathrm{V} 1$, but how do these signals influence responses across the hierarchy of mouse visual areas? For example, does the spatial modulation of visual responses begin in cortex or are they inherited from LGN thalamic inputs? Recordings in freely-moving animals suggest that spatial signals might also be present in the LGN [43]. However, LGN afferents in V1, recorded by expressing a calcium indicator in LGN neurons and imaging boutons in V1, show negligible spatial modulation of responses in a virtual reality environment [16]. Recordings from six different higher visual areas, $L M, A L, R L, A, A M$ and $P M$, revealed that spatial modulation is ubiquitous among the observed cortical visual areas [16]. Therefore, this suggests the modulation of visual responses by spatial signals starts from $\mathrm{V} 1$ and persist across the higher visual areas.

As described above, there is growing evidence of spatial signals in visual areas of the brain. In parallel, there is also a better understanding of the integration of visual signals with 
idiothetic signals (signals that relate to physical distance travelled) across a range of navigational brain regions: retrosplenial cortex (RSC) [18,44], medial enthorhinal cortex (MEC) [45-48] and CA1 [46,49-52]. Given the increased understanding of how individual areas function during navigation (especially in virtual environments), we can now consider how information flows between the visual and navigational regions of the brain.

\section{Two-streams of processing for navigation}

While we have discussed how navigation alters activity in visual areas, how do navigational regions of the brain build a representation of the environment - like the cognitive map in the hippocampus [53]? To create a cognitive map, the hippocampus needs to use available sensory information. This sensory information includes visual information, especially in virtual environments where spatial position is defined based on visual cues. So, how does information about visual cues flow from visual areas to the hippocampus?

The primary routes through which the hippocampus receives cortical inputs are the entorhinal cortices: the medial entorhinal cortex (MEC) and the lateral entorhinal cortex (LEC). The MEC contains cells that encode features related to self-motion, including grid cells [54,55], speed cells [56], and head-direction [55,57]. The LEC instead has neurons that encode object related information, including object vector cells (cells that are active when the animal is at a certain distance and direction from an object) [58], or trace cells (cells that fire in locations where an object was previously present) [59]. Based on the specialisation in MEC and LEC, it has been suggested [60] that these areas (that receive multimodal inputs from various sensory and motor regions) represent the end-points of the what and where streams of visual processing, which are then integrated at the hippocampus (Figure 3). Functionally, self-motion related information would benefit from optic flow related signals. One the other hand, the representation of landmarks and objects would need information related to visual recognition such as fine features. Therefore, visual information relevant for navigation can be broadly segregated into one stream for object and landmark features, and a second stream for optic flow signals (Figure 3 ). This segregation of information is potentially useful also for non-navigational functions. For instance, information about selfmotion can be used for motor control, while information regarding objects and landmarks can be used for visual recognition.

\section{Two-streams based on visual field coverage - central and peripheral visual streams}

Here, we suggest a variation of the two-stream hypothesis based on visual field coverage: one stream includes areas processing information related to objects and landmarks within the central visual field, while the second stream is biased to process movement related information from the peripheral visual field. This hypothesis is inspired by two key observations: the distribution of navigationally relevant information across the visual scene, and biases of visual field coverage in higher visual areas (Figure 4).

The first observation is a bias in visual information across the visual field during navigation. Most large movements while navigating tend to be forward translations (moving straight ahead), interspersed with head and body rotations to orient oneself towards a target. The 
rotations are likely controlled by superior collicular circuits [61]. Forward translations generate an expanding optic flow pattern going outward from the target landmark. This would result in motion vectors whose amplitude increases as we move further away from the target, are maximal on the side and then reduce again towards a point behind the animal [62]. Therefore, the motion vectors are small in the central part of the visual field and larger in most of the periphery visual field for forward translation (Figure 4A-B). In conjunction, natural scenes tend to have more features near the horizon and in the lower part of the visual field. The lower field also has features that are closer to the observer, and as we go skywards into the upper visual field, features represent aspects that are more distant and sparser (Figure 4C). This suggests that the most information regarding movement or speed is present in the lower periphery, while the central visual field is likely to have the landmarks that the subject is interested in and heading towards (Figure 4D). Incidentally, as the binocular region of mouse vision is within it, the central field is also useful in estimating distance to a landmark or object.

The second observation is that individual higher visual areas have biases with regard to coverage of the visual field [25-27]. Visual areas medial to V1 predominantly process information in the far peripheral visual field, while areas lateral to V1 are biased towards the central visual field (Figure 4E-F) [26]. This incomplete representation could be considered a limitation of the rodent visual system. However, it could also be a feature, where areas specialized for certain visual features are biased to view the part of the visual field with the most relevant information. For example, optic flow might be best processed in the periphery part of the visual field. Thus, the limited visual-field representation of higher visual areas can potentially benefit their corresponding functional specialisation.

The two observations above suggest that areas specialised to observe the lower periphery are better suited to access visual information related to the speed of the animal. Conversely, areas observing the central visual field are better suited to recognising landmarks and obstacles. Therefore, we hypothesize that navigational information is processed along these central and peripheral streams, where the 'central' stream processes landmark and object information, while the 'peripheral' stream processes self-motion signals. This would place areas $L M, L i, A L \& R L$ along the central streams, and areas PM, P, AM \& A along the peripheral streams. Based on the function and the identified areas the central stream is analogous to the ventral stream and the peripheral stream to the dorsal stream.

Based on the hypothesis of two-streams of visual processing for navigation, we can make a couple of predictions on their function. The first prediction is based on the fact that spatial representations in the place cells and head-direction cells are known to be strongly controlled by visual cues (or landmarks) [2-6]. As the hypothesis suggests landmarks and objects are processed by the central stream, it would predict cue-locking is controlled by the central visual stream.

Recordings of place cells and grid cells in virtual environments show that virtual expansion or contraction of the environment, causes similar effects in the representation of distance travelled in the environment $[45,46,51]$. Such effects are likely controlled by self-motion signals including optic flow, which the hypothesis attributes to the peripheral stream. Therefore, we predict that areas along the peripheral stream control the contraction and expansion of representations observed in navigational areas. 
While we have discussed the two-stream hypothesis with regards to how visual information reaches hippocampal structures, information flow is likely to be bidirectional, especially given the presence of spatial signals in visual areas [14-16,43]. While the main output regions from the hippocampal circuits to the cortex are the subiculum and entorhinal cortices - which of them are specifically involved in routing spatial signals to visual areas remains an open question.

\section{Conclusions}

In this article, we reviewed how visual responses are modulated by spatial signals in the primary visual cortex [14,15] and across mouse visual areas [16]. These findings show how visual processing can be different during navigation, and highlight the importance of studying visual coding during navigation. In parallel, virtual reality has also been used to understand how spatial signals are processed across a range of brain areas, including RSC $[18,44]$, MEC [45-48] and CA1 [7,46,49-52]. With this improved expertise, we can now take advantage of virtual reality systems to systematically test various aspects of navigational processing with virtual or neural manipulations, looking at layer based [63], local circuits, or projection specific populations [64-67] to investigate mouse visual processing during navigation.

While the focus of this article has been on the mouse visual system, some of the observations can also relate to other species. For instance, the distribution of visual information across the visual field during navigation would be similar across species (Figure 4A-D). In addition, recent primate studies found a bias in visual field coverage in some higher visual areas: they find specific brain regions that process the far periphery $\left(>50^{\circ}\right)$, based on both electrophysiology in primates [68-70], and fMRI in human subjects [71,72]. Using new display technologies, perhaps future research may yet discover more about visual processing in the far periphery [68], including perceptual differences like those observed between the central and peripheral visual fields in human subjects [73].

Here, we proposed a two-stream hypothesis for processing navigational information that is based on visual field coverage. While this hypothesis predicts certain specialisation of higher visual areas, the distribution of functional properties might not be fully distinct, and there might be overlap between the streams. For example, MEC which is considered to have neurons that represent self-motion related signals, also has a representation of landmarks $[47,48]$. This could either be from receiving inputs from both visual streams, or the presence of mixed representations within the peripheral stream. Regardless of the precise anatomical separation into two-streams, the functional segmentation of navigationally relevant visual information, into landmark and self-motion related signals, can provide an accessible framework to design future experiments.

\section{Acknowledgements}

I thank Mika Diamanti, Sam Solomon and Mai Morimoto for feedback on the manuscript. This work was supported by The Sir Henry Dale Fellowship from the Wellcome Trust and Royal Society (200501), Human Frontier in Science Program (RGY0076/2018), and the Biotechnology and Biological Sciences Research Council (R004765) research grants. 


\section{Figures}

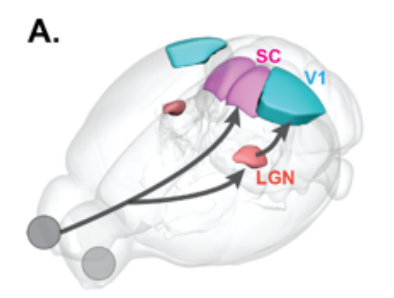

B.

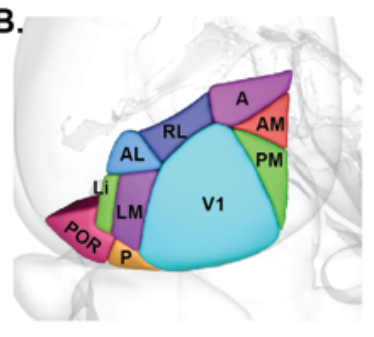

C. Virtual Reality environment

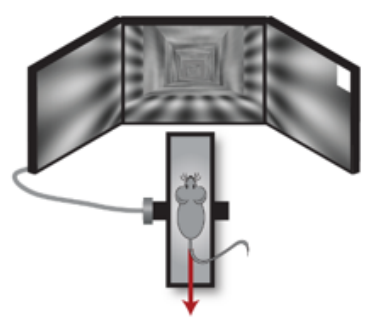

D.

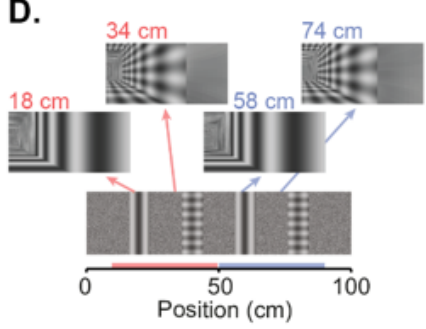

E. Example V1 responses
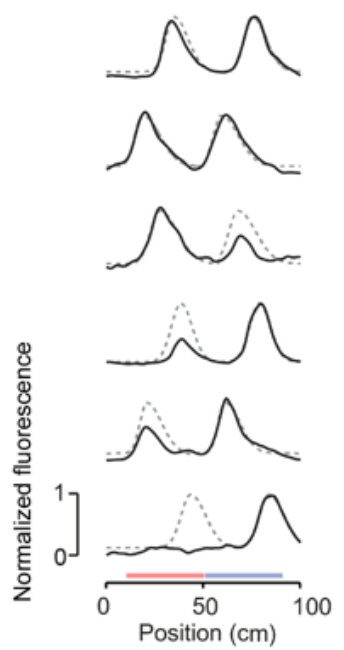

Figure 1. Visual areas in the mouse brain and spatial modulation in the primary visual cortex. A. Early visual areas in mouse brain (LGN: lateral geniculate nucleus; SC: Superior Colliculus; V1: Primary visual cortex). B. Higher visual areas in mouse cortex (P: posterior; POR: postrhinal cortex; LM: lateromedial area; Li: lateral area; AL: anterolateral; RL: rostrolateral; A: anterior; AM: anteriomedial; PM: posteromedial areas). C. The virtual reality environment where the mouse can navigate the virtual environment by running on a styrofoam wheel. $\mathbf{D}$. Design of the virtual environment with two identical segments. Images from half the display are shown from four positions, which include two images from the first segment (at $18 \mathrm{~cm}$ and $34 \mathrm{~cm}$ ) and two from the visually matching positions in the second segment (at $58 \mathrm{~cm}$ and $74 \mathrm{~cm}$ ). E. Response as a function of position of six example neurons recorded from the primary visual cortex. A-B are drawn in [74], based on the Allen common coordinate framework. C-E. Adapted from [14].

A.

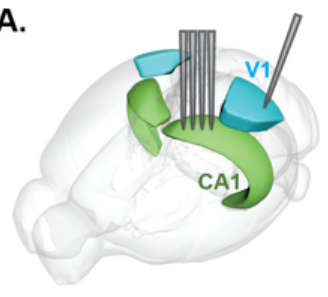

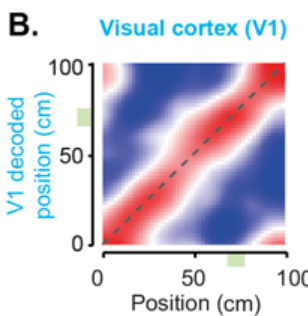

Figure 2. Coherent representation of spatial information in the primary visual cortex and hippocampus.

A. Schematic of simultaneous recordings from the primary visual cortex and CA1 region of the hippocampus using multi-electrode arrays. B. Distribution of decoded position as a function of the actual position of the animal in VR in V1 (left) and CA1 (right). (adapted from [14]). 


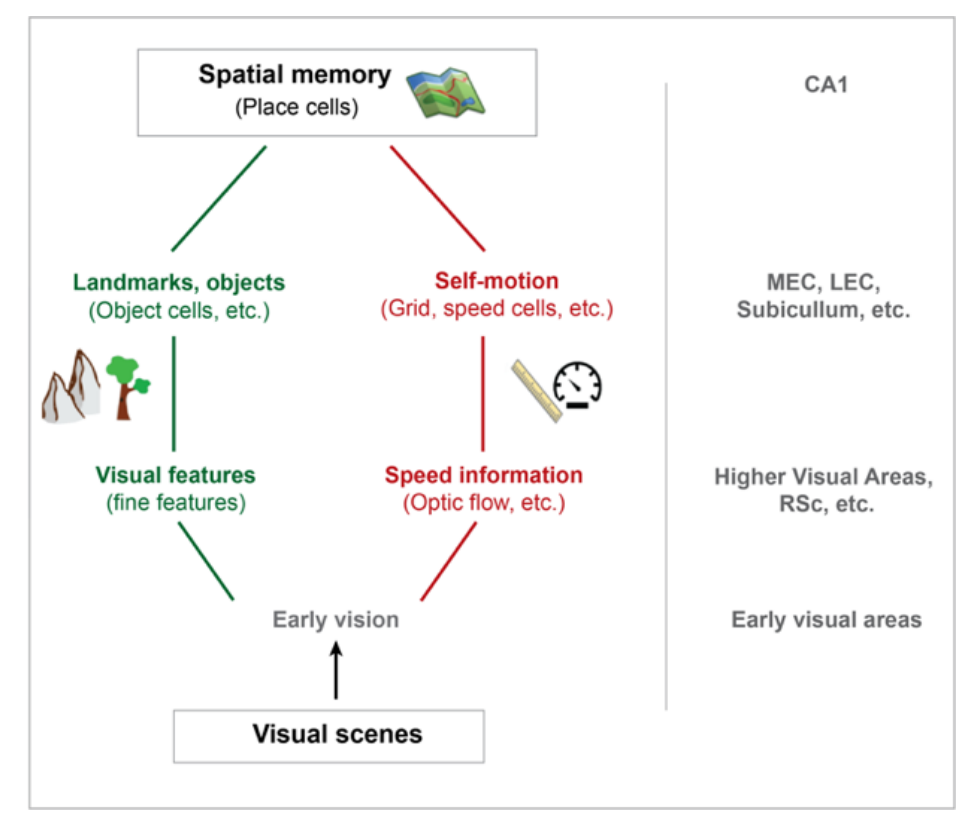

Figure 3: Two hypothesized streams of visual processing for navigation.
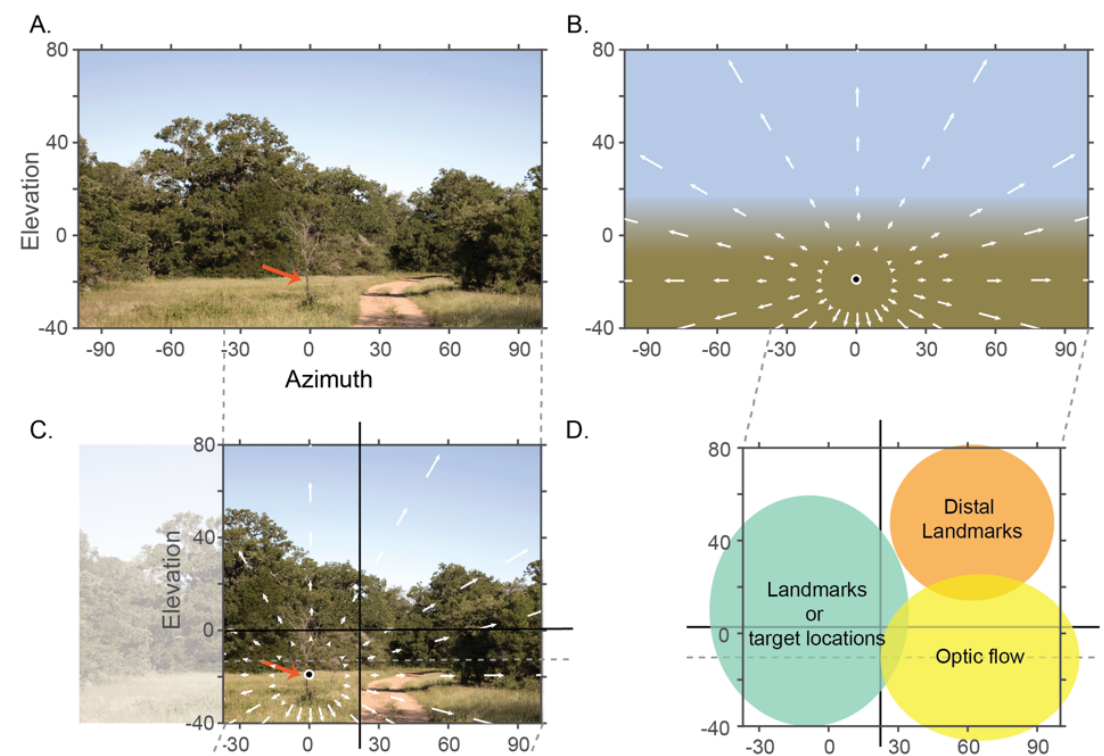

E.

C.
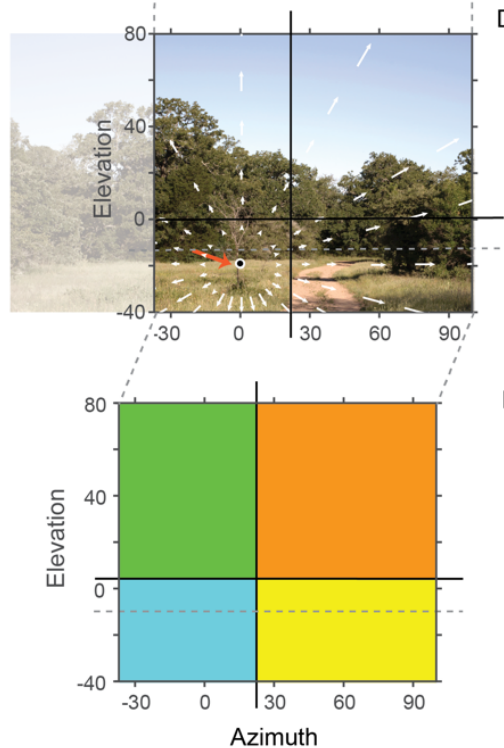

$\mathrm{F}$.

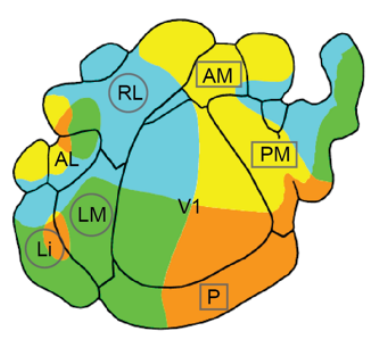

Figure 4: Hypothesized central and peripheral visual streams for navigation. A. Example natural scene [75] The red arrow points to a potential target / landmark location B. Schematic of the optic flow pattern expected for forward translation towards a central target / landmark (based on [76]) C. The overlay of the optic flow vectors on the sample natural image. D. Hypothesized distribution of the visual information at different positions in the visual field (While the chosen natural scene in A,C has no features in the region highlighted 'Distal landmarks', one can expect distant features in landscapes, such as hills, in this region) E-F. Visual field representation across higher 
visual areas. Modified from [26]. Areas hypothesised to be on a central pathway are circled, while those on the peripheral pathway are in a rectangular box.

\section{References}

1. Morris RGM: Spatial localization does not require the presence of local cues. Learn Motiv 1981, 12:239-260.

2. O'Keefe J, Conway DH: Hippocampal place units in the freely moving rat: Why they fire where they fire. Exp Brain Res 1978, 31:573-590.

3. Muller RU, Kubie JL: The effects of changes in the environment on the spatial firing of hippocampal complex-spike cells. $J$ Neurosci 1987, 7:1951-1968.

4. Wiener SI, Taube JS: Head direction cells and the neural mechanisms of spatial orientation. MIT Press; 2005.

5. Muller RU, Ranck JB, Taube JS: Head direction cells: Properties and functional significance. Curr Opin Neurobiol 1996, 6:196-206.

6. Jeffery KJ, O'Keefe JM: Learned interaction of visual and idiothetic cues in the control of place field orientation. Exp Brain Res 1999, 127:151-161.

7. Acharya L, Aghajan ZM, Vuong C, Moore JJ, Mehta MR: Causal Influence of Visual Cues on Hippocampal Directional Selectivity. Cell 2016, 164:197-207.

8. Cao L, Händel B: Walking enhances peripheral visual processing in humans. PLOS Biol 2019, 17:e3000511.

9. Benjamin A V., Wailes-Newson K, Ma-Wyatt A, Baker DH, Wade AR: The effect of locomotion on early visual contrast processing in humans. $J$ Neurosci 2018, 38:3050-3059.

10. Harvey CD, Collman F, Dombeck DA, Tank DW: Intracellular dynamics of hippocampal place cells during virtual navigation. Nature 2009, 461:941-946.

11. Dombeck DA, Harvey CD, Tian L, Looger LL, Tank DW: Functional imaging of hippocampal place cells at cellular resolution during virtual navigation. Nat Neurosci 2010, 13:1433-1440.

12. Drew L: The mouse in the video game. Nature 2019, 567:158-160.

13. Dombeck DA, Reiser MB: Real neuroscience in virtual worlds. Curr Opin Neurobiol 2012, 22:3-10.

14. Saleem AB, Diamanti EM, Fournier J, Harris KD, Carandini M: Coherent encoding of subjective spatial position in visual cortex and hippocampus. Nature 2018, doi:10.1038/s41586-018-0516-1.

15. Fournier J, Saleem AB, Diamanti EM, Wells MJ, Harris KD, Carandini M: Modulation of visual cortex by hippocampal signals. bioRxiv 2019, doi:10.1101/586917.

16. Diamanti EM, Reddy C, Schröder S, Harris KD, Saleem AB, Carandini M: Spatial encoding in the visual pathway arises in cortex and depends on active navigation. bioRxiv 2019, doi:10.1101/832915.

17. Fiser A, Mahringer D, Oyibo HK, Petersen A V, Leinweber M, Keller GB: Experiencedependent spatial expectations in mouse visual cortex. Nat Neurosci 2016, 19:1658-1664.

18. Fischer LF, Soto-Albors RM, Buck F, Harnett MT: Representation of Visual Landmarks in Retrosplenial Cortex. bioRxiv 2019, doi:10.1101/811430.

19. Pakan JMP, Currie SP, Fischer L, Rochefort NL: The Impact of Visual Cues, Reward, and Motor Feedback on the Representation of Behaviorally Relevant Spatial Locations in Primary Visual Cortex. Cell Rep 2018, doi:10.1016/j.celrep.2018.08.010.

20. Pakan JM, Francioni V, Rochefort NL: Action and learning shape the activity of neuronal circuits in the visual cortex. Curr Opin Neurobiol 2018, 52:88-97.

21. Glickfeld LL, Olsen SR: Higher-Order Areas of the Mouse Visual Cortex. Annu Rev Vis Sci 2017, doi:10.1146/annurev-vision-102016-061331.

22. Seabrook TA, Burbridge TJ, Crair MC, Huberman AD: Architecture, Function, and Assembly of the Mouse Visual System. 2017, doi:10.1146/annurev-neuro-071714.

23. Dräger UC: Receptive fields of single cells and topography in mouse visual cortex. J Comp Neurol 1975, 160:269-289.

24. Wang Q, Gao E, Burkhalter A: Gateways of Ventral and Dorsal Streams in Mouse Visual Cortex. J Neurosci 2011, 31:1905-1918. 
25. Garrett ME, Nauhaus I, Marshel JH, Callaway EM, Garrett ME, Marshel JH, Nauhaus I, Garrett ME: Topography and areal organization of mouse visual cortex. J Neurosci 2014, doi:10.1523/JNEUROSCI.1124-14.2014.

26. Zhuang J, Ng L, Williams D, Valley M, Li Y, Garrett M, Waters J: An extended retinotopic map of mouse cortex. Elife 2017, doi:10.7554/eLife.18372.

27. Wang Q, Burkhalter A: Area map of mouse visual cortex. J Comp Neurol 2007, 502:339_ 357.

28. Mishkin M, Ungerleider LG: Contribution of striate inputs to the visuospatial functions of parieto-preoccipital cortex in monkeys. Behav Brain Res 1982, doi:10.1016/01664328(82)90081-X.

29. Milner AD, Goodale MA: Visual pathways to perception and action. Prog Brain Res 1993, doi:10.1016/S0079-6123(08)60379-9.

30. Wang Q, Sporns $\mathrm{O}$, Burkhalter A: Network analysis of corticocortical connections reveals ventral and dorsal processing streams in mouse visual cortex. $J$ Neurosci 2012 , doi:10.1523/JNEUROSCI.6063-11.2012.

31. Andermann ML, Kerlin AM, Roumis DK, Glickfeld LL, Reid RC: Functional specialization of mouse higher visual cortical areas. Neuron 2011, doi:10.1016/j.neuron.2011.11.013.

32. Van Den Bergh G, Zhang B, Arckens L, Chino YM: Receptive-field properties of V1 and V2 neurons in mice and macaque monkeys. J Comp Neurol 2010, doi:10.1002/cne.22321.

33. Marshel JH, Garrett ME, Nauhaus I, Callaway EM: Functional specialization of seven mouse visual cortical areas. Neuron 2011, doi:10.1016/j.neuron.2011.12.004.

34. Murakami T, Matsui T, Ohki K: Functional segregation and development of mouse higher visual areas. J Neurosci 2017, doi:10.1523/JNEUROSCI.0731-17.2017.

35. Smith IT, Townsend LB, Huh R, Zhu H, Smith SL: Stream-dependent development of higher visual cortical areas. Nat Neurosci 2017, doi:10.1038/nn.4469.

36. Juavinett AL, Callaway EM: Pattern and Component Motion Responses in Mouse Visual Cortical Areas. Curr Biol 2015, 25:1759-1764.

37. Sit KK, Goard MJ: Distributed and Retinotopically Asymmetric Processing of Coherent Motion in Mouse Visual Cortex. bioRxiv 2019, doi:10.1101/791905.

38. de Vries SEJ, Lecoq JA, Buice MA, Groblewski PA, Ocker GK, Oliver M, Feng D, Cain N, Ledochowitsch $\mathrm{P}$, Millman D, et al.: A large-scale standardized physiological survey reveals functional organization of the mouse visual cortex. Nat Neurosci 2020, 23:138-151.

39. Ji D, Wilson MA: Coordinated memory replay in the visual cortex and hippocampus during sleep. Nat Neurosci 2007, 10:100-107.

40. Haggerty DC, Ji D: Activities of visual cortical and hippocampal neurons co-fluctuate in freely moving rats during spatial behavior. Elife 2015, 4.

41. Saleem AB, Ayaz A, Jeffery KJ, Harris KD, Carandini M: Integration of visual motion and locomotion in mouse visual cortex. Nat Neurosci 2013, 16:1864-1869.

42. Pakan JMP, Currie SP, Fischer L, Rochefort Correspondence NL: The Impact of Visual Cues, Reward, and Motor Feedback on the Representation of Behaviorally Relevant Spatial Locations in Primary Visual Cortex. 2018, doi:10.1016/j.celrep.2018.08.010.

43. Hok V, Jacob P-Y, Bordiga P, Poucet B, Save E: A spatial code in the dorsal lateral geniculate nucleus. bioRxiv 2018, doi:10.1101/473520.

44. Mao D, Kandler S, McNaughton BL, Bonin V: Sparse orthogonal population representation of spatial context in the retrosplenial cortex. Nat Commun 2017, 8:243.

45. Campbell MG, Ocko SA, Mallory CS, Low IIC, Ganguli S, Giocomo LM: Principles governing the integration of landmark and self-motion cues in entorhinal cortical codes for navigation. Nat Neurosci 2018, 21:1096-1106.

46. Chen G, Lu Y, King JA, Cacucci F, Burgess N: Differential influences of environment and self-motion on place and grid cell firing. Nat Commun 2019, doi:10.1038/s41467-01908550-1.

47. Kinkhabwala A, Gu Y, Aronov D, Tank DW: Visual cue-related activity of cells in the medial entorhinal cortex during navigation in virtual reality. bioRxiv 2018, doi:10.1101/453787.

48. Casali G, Shipley S, Dowell C, Hayman R, Barry C: Entorhinal Neurons Exhibit Cue 
Locking in Rodent VR. Front Cell Neurosci 2019, 12.

49. Chen G, King JA, Burgess N, O'Keefe J: How vision and movement combine in the hippocampal place code. Proc Natl Acad Sci U S A 2013, 110:378-383.

50. Ravassard P, Kees A, Willers B, Ho D, Aharoni D, Cushman J, Aghajan ZM, Mehta MR: Multisensory Control of Hippocampal Spatiotemporal Selectivity. Science (80- ) 2013, 340:1342-1346.

51. Jayakumar RP, Madhav MS, Savelli F, Blair HT, Cowan NJ, Knierim JJ: Recalibration of path integration in hippocampal place cells. Nature 2019, 566:533-537.

52. Cushman JD, Aharoni DB, Willers B, Ravassard P, Kees A, Vuong C, Popeney B, Arisaka K, Mehta MR: Multisensory control of multimodal behavior: Do the legs know what the tongue is doing? PLoS One 2013, 8.

53. O'Keefe J, Dostrovsky J: The hippocampus as a spatial map. Preliminary evidence from unit activity in the freely-moving rat. Brain Res 1971, 34:171-175.

54. Fyhn M, Molden S, Witter MP, Moser EI, Moser MB: Spatial representation in the entorhinal cortex. Science (80-) 2004, 305:1258-1264.

55. Moser EI, Kropff E, Moser M-B: Place Cells, Grid Cells, and the Brain's Spatial Representation System. Annu Rev Neurosci 2008, 31:69-89.

56. Kropff E, Carmichael JE, Moser MB, Moser EI: Speed cells in the medial entorhinal cortex. Nature 2015, 523:419-424.

57. Sargolini F, Fyhn M, Hafting T, McNaughton BL, Witter MP, Moser M-B, Moser EI: Conjunctive Representation of Position, Direction, and Velocity in Entorhinal Cortex. Science (80-) 2006, 312.

58. Deshmukh SS, Knierim JJ: Representation of non-spatial and spatial information in the lateral entorhinal cortex. Front Behav Neurosci 2011, 5:69.

59. Tsao A, Moser MB, Moser EI: Traces of experience in the lateral entorhinal cortex. Curr Biol 2013, 23:399-405.

60. Knierim JJ, Neunuebel JP, Deshmukh SS: Functional correlates of the lateral and medial entorhinal cortex: objects, path integration and local-global reference frames. Philos Trans R Soc Lond B Biol Sci 2014, 369:20130369.

61. Masullo L, Mariotti L, Alexandre N, Freire-Pritchett P, Boulanger J, Tripodi M: Genetically Defined Functional Modules for Spatial Orienting in the Mouse Superior Colliculus. Curr Biol 2019, doi:10.1016/j.cub.2019.07.083.

62. Sabbah S, Gemmer JA, Bhatia-Lin A, Manoff G, Castro G, Siegel JK, Jeffery N, Berson DM: A retinal code for motion along the gravitational and body axes. Nature 2017, doi:10.1038/nature22818.

63. Ayaz A, Stäuble A, Hamada M, Wulf MA, Saleem AB, Helmchen F: Layer-specific integration of locomotion and sensory information in mouse barrel cortex. Nat Commun 2019, 10.

64. Yamashita T, Pala A, Pedrido L, Kremer Y, Welker E, Petersen CCH: Membrane potential dynamics of neocortical projection neurons driving target-specific signals. Neuron 2013, 80:1477-1490.

65. Chen JL, Carta S, Soldado-Magraner J, Schneider BL, Helmchen F: Behaviour-dependent recruitment of long-range projection neurons in somatosensory cortex. Nature 2013, 499:336-340.

66. Glickfeld LL, Andermann ML, Bonin V, Reid RC: Cortico-cortical projections in mouse visual cortex are functionally target specific. Nat Neurosci 2013, doi:10.1038/nn.3300.

67. Lur G, Vinck MA, Tang L, Cardin JA, Higley MJ: Projection-Specific Visual Feature Encoding by Layer 5 Cortical Subnetworks. Cell Rep 2016, 14:2538-2545.

68. Yu HH, Chaplin TA, Rosa MGP: Representation of central and peripheral vision in the primate cerebral cortex: Insights from studies of the marmoset brain. Neurosci Res 2015, 93:47-61.

69. Angelucci A, Rosa MGP: Resolving the organization of the third tier visual cortex in primates: A hypothesis-based approach. Vis Neurosci 2015, 32.

70. Palmer SM, Rosa MGP: A distinct anatomical network of cortical areas for analysis of motion in far peripheral vision. Eur J Neurosci 2006, 24:2389-2405. 
71. Zhu Q, Vanduffel W: Submillimeter fMRI reveals a layout of dorsal visual cortex in macaques, remarkably similar to New World monkeys. Proc Natl Acad Sci U S A 2019, 116:2306-2311.

72. Tamietto M, Leopold DA: Visual Cortex: The Eccentric Area Prostriata in the Human Brain. Curr Biol 2018, 28:R17-R19.

73. Zhaoping L: Feedback from higher to lower visual areas for visual recognition may be weaker in the periphery: Glimpses from the perception of brief dichoptic stimuli. Vision Res 2017, doi:10.1016/j.visres.2017.05.002.

74. Bezgin G, Reid AT, Schubert D, Kötter R: Matching spatial with ontological brain regions using java tools for visualization, database access, and integrated data analysis. Neuroinformatics 2009, doi:10.1007/s12021-008-9039-5.

75. Geisler WS, Perry JS: Statistics for optimal point prediction in natural images. $J$ Vis 2011, doi:10.1167/11.12.14.

76. Gibson J: The perception of the visual world. 1950,

1. **(Saleem et at, 2018)

This article shows clear evidence that responses of neurons in the primary visual cortex are modulated by spatial position, and the modulations are correlated with spatial signals in the hippocampus.

2. *(Fournier et al, 2019)

By manipulating the gain of a virtual reality environment, this study shows that self-motion signals that are known to influence hippocampal signals also modulate visual signals. It also shows the dependence of responses in the primary visual cortex on hippocampal theta oscillations.

3. *(Diamanti et al, 2019)

This study investigated the influence of spatial signals along the visual hierarchy, and suggests that these signals are not present in the lateral geniculate nucleus inputs to the primary visual cortex, and spatial modulation is present in the primary visual cortex and higher visual areas.

4. *(Fiser et al, 2016)

This study finds that a small fraction of neurons in the superficial layers of the primary visual cortex contain signals that are predictive of upcoming stimuli, which is also present in the neurons of the anterior cingulate cortex projecting to V1. They also find a small number of neurons that respond to the omission of a stimulus presentation.

5. **(Glickfeld \& Olsen, 2017)

An excellent review of the structural and functional properties of mouse visual areas. It also discusses the separation of area between putative dorsal and ventral streams of processing for visual information.

6. **(Zhuang et al, 2017)

An analysis of receptive field coverage of mouse visual areas. This article highlights the biases present in various higher visual areas, i.e. that they are responsive to limited regions of the visual field.

7. **(Pakan et al)

This article shows how the spatial position of reward is represented in the primary visual cortex during learning of a visual navigation task. They find an increased representation of reward position with learning. Interestingly, they find that the reward position is encded even in the absence of visual cues reward position using self-motion cues.

8. **(de Vries et al)

An impressive resource of functional response of mouse higher visual areas. One interesting finding is that many cells in visual areas respond uniquely to specific stimulus categories, such as gratings, natural scenes or natural movies.

9. *(Haggerty et al)

One of the first studies of simultaneous recordings of visual cortical neurons and hippocampal neurons in freely moving animals. This article shows that visual and hippocampal neurons 
with similar response fields co-fluctuate, i.e. neurons in both areas fire action potentials in the early part of the field, or the later part of the field together on each run through the field.

10. *(Sabbah et al.)

This study investigated the topographic distribution of direction selective cells of the retina, and compared them to optic flow patterns of self-motion. They found that the distribution of direction selective cells are optimally tuned to represent translational movements. 